\title{
Scoping Review
}

\section{What is known about human milk bank donors around the world: a systematic scoping review}

\author{
Bruna Gutierrez dos Santos and Maryanne T Perrin* (1) \\ Nutrition Department, University of North Carolina Greensboro, 319 College Avenue, 318 Stone Building, \\ Greensboro, NC 27412, USA
}

Submitted 27 April 2021: Final revision received 6 August 2021: Accepted 9 September 2021: First published online 13 September 2021

\begin{abstract}
Objective: The WHO recommends that low birth weight infants receive donor human milk (DHM) when mother's milk is not available. Systematic reviews have been published regarding clinical outcomes of infants receiving DHM, as well as the impact of pasteurisation on the composition of DHM; however, information about milk bank donors has not been systematically assessed.

Design: We conducted a systematic scoping review of original research articles about milk bank donors published before August 2020.

Setting: Globally.

Participants: Donors to milk banks.

Results: A total of twenty-eight studies were included across a variety of geographies: the USA ( $n$ 8), Brazil ( $n 7)$, Spain ( $n$ 4), India ( $n$ 2), and single studies in France, Norway, Poland, Italy, Taiwan, Korea and China. Study variables were grouped into six main categories: Donor Demographics ( $n$ 19), Clinical Characteristics ( $n$ 20), Donor Experiences ( $n$ 16), Donation Patterns ( $n$ 16), Lifestyle Characteristics ( $n$ 4) and Lactation/Breast-feeding History ( $n$ 8). Some demographic characteristics were commonly reported across regions, while other, including gender and race, were infrequently explored. Factors that might influence the composition of DHM, including birth timing (term or pre-term), milk type (colostrum, transition or mature) and maternal diet were not regularly studied. Other gaps in the literature included (1) donors' motivations and barriers to donation, (2) lactation and breast-feeding history, including factors that influence donors to pump and amass surplus milk, and (3) donation patterns, including whether donors are also selling milk to corporations or sharing milk with peers. Conclusion: What is known about milk bank donors in different geographies is often limited to a single study, with heterogeneity in the variables reported.
\end{abstract}

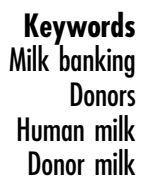

The WHO recommends that low birth weight infants receive donor human milk (DHM) when mother's own milk is not available due to evidence that it decrease the risk of necrotising enterocolitis ${ }^{(1,2)}$. Globally, DHM is typically produced by country-level milk banking networks that serve as a conduit between the recipient infants and the donors who provide the milk ${ }^{(3-5)}$. Although the recommended recipient for DHM is primarily the pre-term infant $^{(2,6)}$, a recent review reported that DHM is also being used in other populations including healthy term infants and term infants with health risks. A 2020 report from a
Virtual Communication Network of global milk banking leaders estimated that at least 800000 infants receive DHM around the world annually ${ }^{(7,8)}$.

To ensure the quality and safety of DHM, human milk banks use similar hazard analysis and critical control points, where protocols are used in every step of the process, from donors screening until milk distribution ${ }^{(9)}$. Holder pasteurisation is the main processing technique used in milk banks, and although it inactivates virus such as HIV and cytomegalovirus, it also alters the milk composition ${ }^{(10)}$. A recent review found over forty studies that had evaluated the impact of Holder 
pasteurisation on DHM, suggesting that there is a growing body of knowledge about this technique ${ }^{(10)}$.

While there are multiple reviews on DHM recipients and milk banking processes, the donors to milk banks have not been systematically studied. A recent report by the WHO noted that the motivations behind donating human milk remain under-researched ${ }^{(11)}$. Other information about milk bank donors may provide important insights regarding donor recruitment and the nutritional care of infants receiving DHM. For example, a donor's birth type (term $v$. pre-term) and milk type (colostrum, transition and mature) could influence the composition of the milk being collected by the milk banks ${ }^{(12)}$. Therefore, the aim of this review is to explore what is currently known about human milk bank donors globally and identify gaps for future research.

\section{Methods}

A systematic scoping review was conducted to investigate what is known about milk bank donors. The objective of a scoping review is to map and summarise the information available for a research topic and to identify gaps where more research is needed ${ }^{(13)}$. The Preferred Reporting Items for Systematic Reviews and Meta-analysis (PRISMA) guidelines were used to guide this review. The databases used to identify original research articles were PubMed and Scopus. Search terms utilised for both databases included 'Milk bank*' AND 'donors' NOT (composition OR pasteuri* OR nutri*). Additional studies were located by hand-reviewing bibliographies of the studies identified through the primary search.

Original research articles about milk bank donors that were published before August 2020 were included in this review. Studies were excluded if they were (1) about donor milk composition and/or pasteurisation only, (2) about infant feeding practices and/or infant nutrition only, (3) in languages that were not English, (4) not original articles or (5) not about milk bank donors (e.g. peer-milk sharing only). Two researchers (BGS and MTP) independently evaluated all study titles, abstracts, and full papers for exclusion or inclusion criteria, and differences were resolved after each review step by discussion.

Included studies were independently abstracted by two researchers (BGS and MTP) into a Microsoft Excel spreadsheet for the following information: study location, study design, study population, study objectives, data collection methods, variables related to milk bank donors, results and funding source. Studies that used multiple years of milk bank donor data were classified as semi-longitudinal study design, since some donors may have appeared more than once in data that spanned several years. Abstracted data were reviewed by two researchers (BGS and MTP) and discrepancies were resolved by discussion. Demographic data from one study combined donor and non-donor information and could not be interpreted; therefore, these demographic data were not reported in the results.

To organise study variables, an iterative process was used by two researchers working together to develop and refine a classification system of main categories and sub-categories for study variables. Categories and subcategories used to classify variables included (1) Donor Demographics (Demographics) which included Age, Marital Status, Race-Ethnicity, Education, and Employment Status, (2) Donor Clinical Characteristics (Clinical) which included Birth History (e.g. number of children, parity, delivery term, neonatal intensive care unit (NICU) admissions), Diseases (e.g. donor health conditions) and Prenatal Care, (3) Donor Lifestyle Characteristics (Lifestyle) which included Diet, Exercise, Legal Drug Use (e.g. nicotine, caffeine, and alcohol) and Illegal Drug Use, (4) Lactation and Breastfeeding Experience (Breast-feeding) which included Breastfeeding History (e.g. breast-feeding experience and problems), Clinical Support, Milk Expression Practices, and Beliefs About the Value of Milk, (5) Donor Experience and Beliefs (Experience/Beliefs) included Reasons/Enablers for Donation, Barriers for Donation and Donor Identity and (6) Donation Patterns (Patterns) included Donation Volume, Donor Type (first-time or repeat), Milk Type (colostrum - 0-7 d, transition milk - 7-21 d, mature milk over $21 \mathrm{~d})^{(14)}$, and Donation Duration.

The primary source of bias considered was selection bias, if donors included in a study were potentially not representative of the broader donor population. Studies were identified as possibly having selection bias if they did not discuss participant selection, had low participation rate (below $60 \%)^{(15)}$ or included a limited sampling frame (e.g. only bereaved donors, only donors active on social media). Selection bias was evaluated independently by two researchers and discrepancies were resolved by discussion.

\section{Results}

A total of 181 studies were identified through Scopus, 84 through PubMed and 8 through hand-review of bibliographies (Fig. 1). After excluding duplicates ( $n$ 70), a total of 203 studies were screened. After a review of abstracts and titles, 154 articles were excluded leaving 49 articles for full-text review. Twenty-one studies were excluded after full-text review leaving twenty-eight studies in this scoping review about human milk bank donors ${ }^{(16-43)}$.

Studies in this systematic review were published between 2003 and 2020 (Table 1) and included 2 to 4000 donors. Eight studies were conducted in the USA, seven in Brazil, four in Spain, two in India, and individual studies were conducted in France, Norway, Poland, Italy, Taiwan, Korea and China. A qualitative design was used 


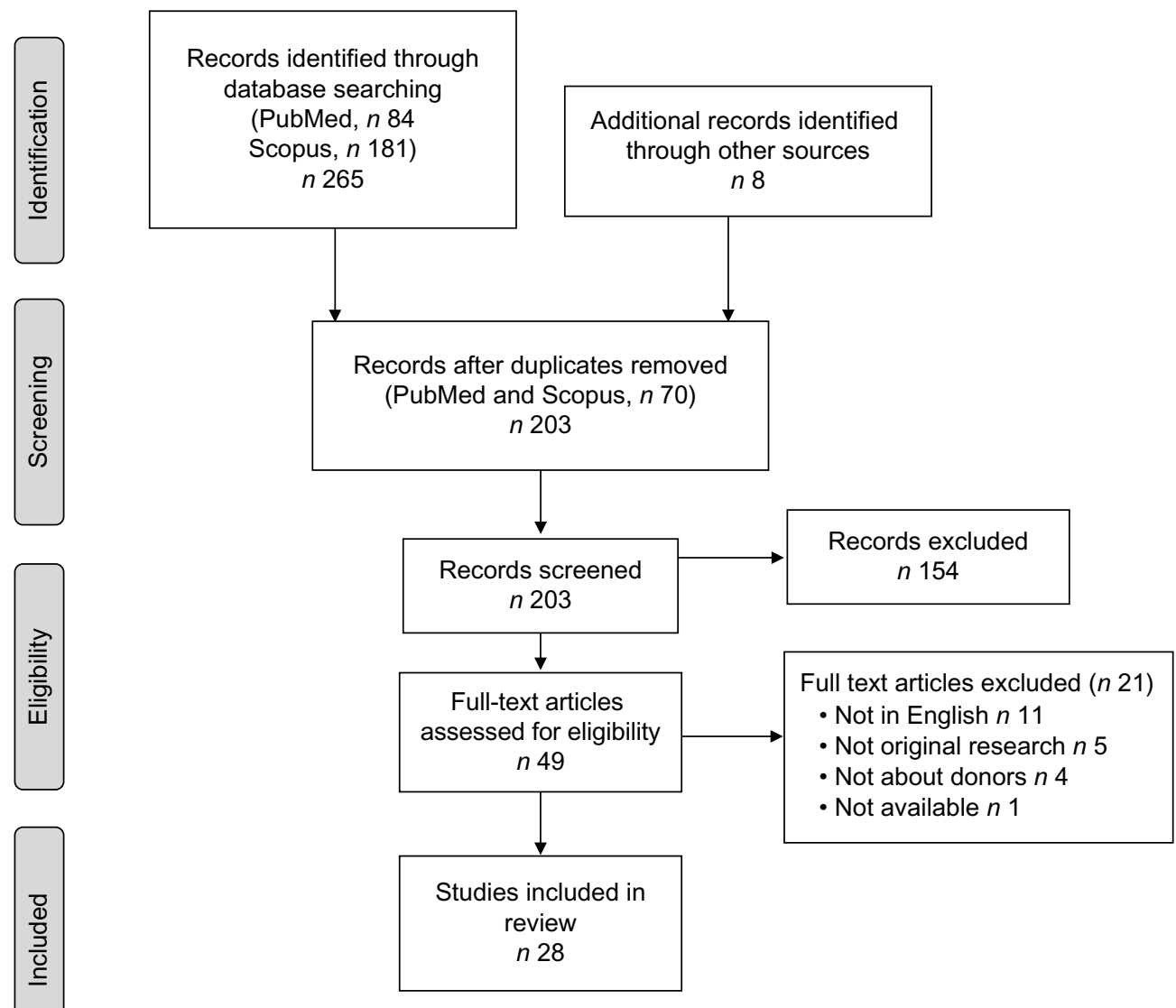

Fig. 1 Flow diagram of the literature search process used to identify studies using the Preferred Reporting Items for Systematic Reviews and Meta-Analyses (PRISMA) checklist

in eight studies, which allows for rich exploration of the donors' lived experiences. Qualitative studies were predominantly conducted in the USA and had a small sample size (2-21 donors and 80-107 online testimonials or images). Data collection methods used in the studies included interviews, questionnaires, chart reviews and online content analysis. In most of the studies, donors were recruited from a single milk bank ( $n$ 16). Ten studies (36\%) presented possible selection bias (Table 1). The number of studies reporting variable types included (1) Donor Demographics ( $n$ 19; Table 2), Clinical Characteristics ( $n$ 20; Table 3), (3) Lifestyle Characteristics ( $n$ 4; Table 4), (4) Lactation/Breast-feeding Experiences ( $n$ 8; Table 5), (5) Donor Experiences ( $n$ 16; Table 6) and (6) Donation Patterns ( $n$ 16; Table 7 ).

\section{Discussion}

Despite reports that there are now over 600 milk banks operating around the world ${ }^{(44)}$, and over 800000 infants annually who receive $\mathrm{DHM}^{(7)}$, studies about milk bank donors are often limited to a single study per geography with significant heterogeneity in the variables reported.

\section{Donor demographics}

Age was the most commonly reported demographic variable, with some initial geographic differences observed. Specifically, donors were predominantly in their early- to mid-twenties in Brazil and India (based on mean donor age or prevalence of donors by age group) ${ }^{(20,21,23,32,34,42)}$, while donors were predominantly in their early-thirties in France, Korea, Norway, Poland, Spain, Taiwan and the USA $^{(16-18,25,27-31,33)}$. There were also geographic differences in education levels among donors, with studies conducted in Brazil reporting that the majority of donors were not college-educated compared to mostly college-educated donors in China, Norway, Spain, Taiwan and the USA $^{(17,19,20,23,25,25,30,39)}$. Across all geographies, donors were predominantly married or living with a partner ${ }^{(16,18-20,30,32,36)}$. Limited information was available on race-ethnicity ${ }^{(18,23,36)}$. No information was collected about gender in any of the studies, suggesting that donor gender may have been assumed in prior research. While this scoping 
Table 1 Summary of studies included in the systematic scoping review of human milk bank donors

\begin{tabular}{|c|c|c|c|c|c|c|c|c|}
\hline Year & Author & $\begin{array}{l}\text { Study } \\
\text { location }\end{array}$ & Study objectives & Population studied & Study design & $\begin{array}{l}\text { Data collection } \\
\text { method }\end{array}$ & $\begin{array}{l}\text { Potential } \\
\text { selection bias }\end{array}$ & Funding source \\
\hline 2003 & Azema $^{(16)}$ & France & $\begin{array}{l}\text { Examine characteristics of donors and } \\
\text { attitudes towards donation }\end{array}$ & $\begin{array}{l}\text { Donors to eight milk banks } \\
\text { ( } n \text { 103) }\end{array}$ & Cross-sectional & Questionnaire & & Information not available \\
\hline 2004 & Lindemann ${ }^{(17)}$ & Norway & $\begin{array}{l}\text { Evaluate donor characteristics and } \\
\text { donation patterns }\end{array}$ & $\begin{array}{l}\text { Donors to a single milk bank in } \\
2001(n 69)\end{array}$ & Not identified & Not identified & & Information not available \\
\hline 2007 & Osbaldiston ${ }^{(18)}$ & USA & $\begin{array}{l}\text { Compare donors and non-donors } \\
\text { characteristics, experiences, motives } \\
\text { and barriers to donation, and the } \\
\text { relationship between donation } \\
\text { experience and amount of milk donated }\end{array}$ & $\begin{array}{l}\text { Donors to a single milk bank } \\
(n 87) \text { and non-donor controls } \\
(n 19)\end{array}$ & Case-control & $\begin{array}{l}\text { Telephone survey } \\
\text { that included } \\
\text { VFI, PANAS, } \\
\text { scale } \\
\text { questions; } \\
\text { chart review }\end{array}$ & Possible & Information not available \\
\hline 2008 & Thomaz $^{(19)}$ & Brazil & $\begin{array}{l}\text { Identify factors that influenced or } \\
\text { motivated donations }\end{array}$ & $\begin{array}{l}\text { Donors to three milk banks } \\
(n 737)\end{array}$ & Cross-sectional & Questionnaire & & Information not available \\
\hline 2009 & Alencar ${ }^{(20)}$ & Brazil & $\begin{array}{l}\text { Describe the behaviour, beliefs and } \\
\text { feelings behind the donations }\end{array}$ & Donors to two milk banks ( $n$ 36) & Cross-sectional & $\begin{array}{l}\text { Structured and } \\
\text { semi-structured } \\
\text { face-to-face } \\
\text { interviews }\end{array}$ & & Information not available \\
\hline 2010 & Alencar ${ }^{(21)}$ & Brazil & $\begin{array}{l}\text { Characterise the behaviour of donation } \\
\text { and formal/informal support }\end{array}$ & Donors to two milk banks ( $n$ 36) & Cross-sectional & $\begin{array}{l}\text { Structured and } \\
\text { semi-structured } \\
\text { face-to-face } \\
\text { interviews }\end{array}$ & & Information not available \\
\hline 2010 & Cohen ${ }^{(22)}$ & USA & $\begin{array}{l}\text { Estimate the seroprevalence of hepatitis B } \\
\text { and C, syphilis, HTLV-1 and } 2 \text { and HIV }\end{array}$ & $\begin{array}{l}\text { Potential donors to a single milk } \\
\text { bank from } 2000 \text { to } 2005 \\
(n \text { 1091) }\end{array}$ & Semi-Iongitudinal & Chart review & & Information not available \\
\hline 2010 & Koyashiki(23) & Brazil & $\begin{array}{l}\text { Evaluate the degree of exposure to lead of } \\
\text { donors }\end{array}$ & $\begin{array}{l}\text { Donors to a single milk bank } \\
\text { ( }(\text { 92) }\end{array}$ & Cross-sectional & $\begin{array}{l}\text { Face-to-face } \\
\text { interview, } \\
\text { questionnaire, } \\
\text { milk sample, } \\
\text { blood sample }\end{array}$ & & Information not available \\
\hline 2012 & Welborn ${ }^{(24)}$ & USA & $\begin{array}{l}\text { Examine the role of milk donation in the } \\
\text { grieving process }\end{array}$ & $\begin{array}{l}\text { Bereaved donors to two milk banks } \\
\quad(n 21)\end{array}$ & $\begin{array}{l}\text { Qualitative, } \\
\text { phenomenological }\end{array}$ & $\begin{array}{l}\text { Semi-structured } \\
\text { face-to-face } \\
\text { and web-based } \\
\text { interviews }\end{array}$ & Possible & No funding obtained \\
\hline 2013 & Chang $^{(25)}$ & Taiwan & $\begin{array}{l}\text { Evaluate donor characteristics and } \\
\text { donation patterns }\end{array}$ & $\begin{array}{l}\text { Donors to a single milk bank from } \\
\text { 2005-2010 }(n \text { 816) }\end{array}$ & Semi-longitudinal & Chart review & & Information not available \\
\hline 2013 & Pineau(26) & USA & $\begin{array}{l}\text { Describe how intensive motherhood and } \\
\text { social class influence milk donations }\end{array}$ & $\begin{array}{l}\text { Donors to a single milk bank } \\
(n 19), \text { including sixteen middle/ } \\
\text { upper income and three WIC } \\
\text { recipients }\end{array}$ & Qualitative & $\begin{array}{l}\text { Face-to-face and } \\
\text { telephone } \\
\text { interviews }\end{array}$ & Possible & Information not available \\
\hline 2014 & Escuder-Vieco(27) & Spain & $\begin{array}{l}\text { Validate the health questionnaire with } \\
\text { respect to the presence of illegal drugs, } \\
\text { nicotine and caffeine in donor milk }\end{array}$ & $\begin{array}{l}\text { Donors to a single milk bank } \\
(n 63)\end{array}$ & Cross-sectional & $\begin{array}{l}\text { Questionnaire } \\
\text { and milk } \\
\text { samples }\end{array}$ & & Spanish Health Research Funding \\
\hline 2014 & Sierra-Colomina ${ }^{(28)}$ & Spain & $\begin{array}{l}\text { Compare the donors social and } \\
\text { demographic characteristics with the } \\
\text { volume of milk donated }\end{array}$ & $\begin{array}{l}\text { Donors to a single milk bank from } \\
\text { 2009-2013 }(n \text { 391) }\end{array}$ & Semi-longitudinal & $\begin{array}{l}\text { Questionnaire } \\
\text { and chart } \\
\text { review }\end{array}$ & & $\begin{array}{l}\text { SAMID (Spanish Collaborative Maternal } \\
\text { and Children and Development) } \\
\text { Research Network }\end{array}$ \\
\hline 2015 & Machado ${ }^{(30)}$ & Spain & $\begin{array}{l}\text { Describe experiences, beliefs, motivations } \\
\text { and difficulties of donations }\end{array}$ & $\begin{array}{l}\text { Donors to a single milk bank } \\
(n 7)\end{array}$ & $\begin{array}{l}\text { Qualitative } \\
\text { phenomenological }\end{array}$ & $\begin{array}{l}\text { Semi-structured } \\
\text { interviews }\end{array}$ & Possible & Information not available \\
\hline 2016 & Escuder-Vieco(29) & Spain & $\begin{array}{l}\text { Determine levels of illegal drugs, nicotine } \\
\text { and caffeine in hair and breast milk }\end{array}$ & $\begin{array}{l}\text { Donors to a single milk bank } \\
(n 36)\end{array}$ & Cross-sectional & $\begin{array}{l}\text { Questionnaire; } \\
\text { hair and milk } \\
\text { samples }\end{array}$ & & Spanish Health Research Funding \\
\hline 2016 & Jang $^{(31)}$ & Korea & $\begin{array}{l}\text { Evaluate donor characteristics and } \\
\text { donation patterns }\end{array}$ & $\begin{array}{l}\text { Donors to a single milk bank from } \\
2008-2015 \text { ( } n \text { 915) }\end{array}$ & Semi-Iongitudinal & $\begin{array}{l}\text { Chart review } \\
\text { using } \\
\text { standardised } \\
\text { form }\end{array}$ & & Information not available \\
\hline
\end{tabular}




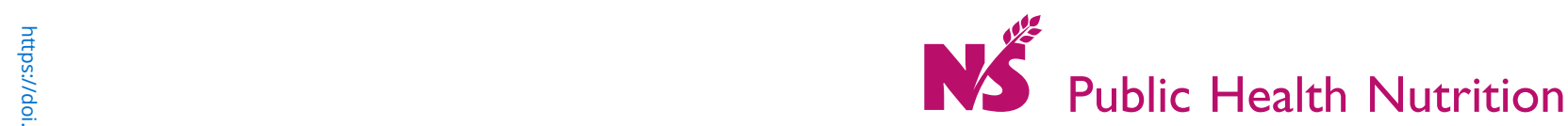

Table 1 Continued

\begin{tabular}{|c|c|c|c|c|c|c|c|c|}
\hline Year & Author & $\begin{array}{l}\text { Study } \\
\text { location }\end{array}$ & Study objectives & Population studied & Study design & $\begin{array}{l}\text { Data collection } \\
\text { method }\end{array}$ & $\begin{array}{l}\text { Potential } \\
\text { selection bias }\end{array}$ & Funding source \\
\hline 2016 & Miranda ${ }^{(32)}$ & Brazil & $\begin{array}{l}\text { Investigate milk donor's representations of } \\
\text { the donation experience }\end{array}$ & Donors to a single milk bank ( $n$ 12) & Qualitative & $\begin{array}{l}\text { Semi-structured } \\
\text { interview }\end{array}$ & Possible & Universidade Federal de Ouro Preto \\
\hline 2017 & Barbarska( $^{(33)}$ & Poland & $\begin{array}{l}\text { Evaluate donor characteristics and } \\
\text { donation patterns }\end{array}$ & $\begin{array}{l}\text { Donors to a single milk bank from } \\
\text { 2015-2016 }(n \text { 45) }\end{array}$ & Semi-Iongitudinal & Chart review & & Information not available \\
\hline 2017 & Kupek $^{(34)}$ & Brazil & $\begin{array}{l}\text { Estimate the seroprevalence of HIV, } \\
\text { syphilis and hepatitis B }\end{array}$ & $\begin{array}{l}\text { Prospective donors to a single milk } \\
\text { bank from 2005-2015 ( } n \text { 3513) }\end{array}$ & Semi-Iongitudinal & Chart review & & No funding obtained \\
\hline 2017 & Meneses $^{(35)}$ & Brazil & $\begin{array}{l}\text { Estimate prevalence and factors } \\
\text { associated with donation }\end{array}$ & $\begin{array}{l}\text { Donors to nine milk banks ( } n \text { 51) } \\
\text { and non-donors control }(n \text { 644) }\end{array}$ & Case-control & $\begin{array}{l}\text { Structured } \\
\text { interviews }\end{array}$ & Possible & $\begin{array}{l}\text { Fundação de Amparo à Pesquisa do } \\
\text { Estado do Rio de Janeiro - FAPERJ }\end{array}$ \\
\hline 2018 & Candelaria ${ }^{(36)}$ & USA & $\begin{array}{l}\text { Examine donors' experiences donating to } \\
\text { milk banks }\end{array}$ & $\begin{array}{l}\text { Donors with infants in the NICU } \\
\quad(n 12)\end{array}$ & $\begin{array}{l}\text { Qualitative } \\
\text { phenomenological }\end{array}$ & $\begin{array}{l}\text { Questionnaire } \\
\text { and semi- } \\
\text { structured } \\
\text { face-to-face } \\
\text { interviews }\end{array}$ & Possible & No funding obtained \\
\hline 2018 & Cole $^{(37)}$ & USA & $\begin{array}{l}\text { Examine milk donation in the context of } \\
\text { perinatal palliative care }\end{array}$ & Bereaved donors ( $n$ 2) & Qualitative case study & $\begin{array}{l}\text { Questionnaire } \\
\text { and telephone } \\
\text { interview }\end{array}$ & Possible & No funding obtained \\
\hline 2018 & Quitadamo ${ }^{(38)}$ & Italy & $\begin{array}{l}\text { Describe donation volume by donor } \\
\text { clinical characteristics }\end{array}$ & $\begin{array}{l}\text { Donors to a single milk bank from } \\
\quad 2010-2017(n 659)\end{array}$ & Semi-longitudinal & Chart review & & Information not available \\
\hline 2019 & $\operatorname{Liu}^{(39)}$ & China & $\begin{array}{l}\text { Characterise milk bank donors and } \\
\text { donation patterns }\end{array}$ & $\begin{array}{l}\text { Donors to fourteen milk banks } \\
\text { 2013-2016 ( } n \text { 2680) }\end{array}$ & Semi-longitudinal & Chart review & & $\begin{array}{l}\text { Guangdong provincial commission of } \\
\text { health and family planning } \\
\text { appropriate technology promotion } \\
\text { project (2015-2017 Guangdong) }\end{array}$ \\
\hline 2019 & Oreg $^{(40)}$ & USA & $\begin{array}{l}\text { Explore milk donation in times of loss to } \\
\text { uncover mechanisms liking grief and } \\
\text { loss to philanthropic giving }\end{array}$ & Bereaved donors ( $n 80)$ & $\begin{array}{l}\text { Qualitative } \\
\text { phenomenological }\end{array}$ & $\begin{array}{l}\text { Content analysis } \\
\text { of online } \\
\text { testimonials }\end{array}$ & Possible & Information not available \\
\hline 2019 & Sachdeva ${ }^{(41)}$ & India & Evaluate the status of milk banks & $\begin{array}{l}\text { Donors to sixteen milk banks from } \\
2015 \text { to } 2016 \text { (range } 70-4000 \\
\text { per bank) }\end{array}$ & Semi-Iongitudinal & $\begin{array}{l}\text { Online } \\
\text { questionnaire } \\
\text { and on-site } \\
\text { interview of } \\
\text { milk bank } \\
\text { personnel }\end{array}$ & & $\begin{array}{l}\text { Margaret A. Cargill Philanthropies to } \\
\text { PATH }\end{array}$ \\
\hline 2020 & Nangia ${ }^{(42)}$ & India & $\begin{array}{l}\text { Classify donors by demographics; } \\
\text { determine and compare milk volume } \\
\text { donated by donor classifications. }\end{array}$ & $\begin{array}{l}\text { Donors to a hospital milk bank from } \\
2017-2019 \text { ( } n \text { 1553) }\end{array}$ & Semi-longitudinal & Chart review & & No funding obtained \\
\hline 2020 & Oreg $^{(43)}$ & USA & $\begin{array}{l}\text { Determine characteristics of the milk donor } \\
\text { identity }\end{array}$ & $\begin{array}{l}\text { Donors' online testimonial }(n 95) \\
\text { and images }(n \text { 107) }\end{array}$ & $\begin{array}{l}\text { Qualitative } \\
\text { phenomenological }\end{array}$ & $\begin{array}{l}\text { Content analysis } \\
\text { of online donor } \\
\text { testimonials } \\
\text { and images }\end{array}$ & Possible & Information not available \\
\hline
\end{tabular}


Table 2 Demographic information about milk bank donors

\begin{tabular}{|c|c|c|c|c|}
\hline Sub-category & Country & Year & Subjects & Findings \\
\hline \multirow[t]{18}{*}{ Age (years) } & $\operatorname{Brazil}^{(19)}$ & 2008 & 737 donors & Majority $<25(18 \%<18 ; 41 \% 18$ to 24$)$ \\
\hline & $\operatorname{Brazil}^{(20,21)}$ & 2009,2010 & 36 donors & Ranged from 14 to 33 ; mean age 25 \\
\hline & Brazil $^{(23)}$ & 2010 & 92 donors & Ranged from 16 to 45 ; mean age 21 \\
\hline & Brazil $^{(32)}$ & 2016 & 12 donors & Ranged from 18 to 39 ; mean age 26 \\
\hline & $\operatorname{Brazil}^{(34)}$ & 2017 & 3513 donors & Majority 20 to $35(80 \%)$ \\
\hline & China $^{(39)}$ & 2019 & 2680 donors & Majority 25 to 35 (82\%); mean age 29 \\
\hline & France $^{(16)}$ & 2003 & 103 donors & Ranged from 20 to 42 ; mean age 31 \\
\hline & India $^{(42)}$ & 2020 & 1553 donors & Majority $<25(88 \%)$ \\
\hline & Korea $^{(31)}$ & 2016 & 915 donors & Majority 30 to $39(70 \%)$ \\
\hline & Norway ${ }^{(17)}$ & 2004 & 69 donors & Ranged from 21 to $45 ;$ mean age 34 \\
\hline & Poland(33) & 2017 & 45 donors & Ranged from 23 to 44 ; mean age 32 \\
\hline & Spain ${ }^{(28)}$ & 2014 & 391 donors & Median age of $34 ;$ IQR of $31-36$ \\
\hline & Spain ${ }^{(27)}$ & 2014 & 63 donors & Ranged from 23 to 53 ; mean age 36 \\
\hline & Spain $^{(30)}$ & 2015 & 7 donors & Ranged from 21 to $39 ;$ mean age 32 \\
\hline & Spain ${ }^{(29)}$ & 2016 & 36 donors & Ranged from 24 to 41 ; mean age 34 \\
\hline & Taiwan ${ }^{(25)}$ & 2013 & 816 donors & Ranged from 18 to 45 ; mean age 31 \\
\hline & USA $^{(18)}$ & 2007 & 87 donors & Majority 30-39 (73\%) \\
\hline & USA $^{(36)}$ & 2018 & 12 donors & All $<40(50 \% 21-29 ; 50 \% 30-39)$ \\
\hline \multirow[t]{7}{*}{ Marital status } & $\operatorname{Brazil}^{(19)}$ & 2008 & 737 donors & Single $(54 \%)$ \\
\hline & $\operatorname{Brazil}^{(20)}$ & 2009 & 36 donors & Married or in a partnership (78\%) \\
\hline & $\operatorname{Brazi}^{(32)}$ & 2016 & 12 donors & Married or in a partnership (75\%) \\
\hline & France $^{(16)}$ & 2003 & 103 donors & Married or in a partnership (97\%) \\
\hline & Spain $^{(30)}$ & 2015 & 7 donors & Married $(86 \%)$ \\
\hline & $\mathrm{USA}^{(18)}$ & 2007 & 87 donors & Married $(91 \%)$ \\
\hline & $\mathrm{USA}^{(36)}$ & 2018 & 12 donors & Married $(100 \%)$ \\
\hline \multirow[t]{3}{*}{ Race-ethnicity } & $\operatorname{Brazil}^{(23)}$ & 2010 & 92 donors & White $(72 \%)$ \\
\hline & $\mathrm{USA}^{(18)}$ & 2007 & 87 donors & White $(87 \%)$ \\
\hline & USA(36) & 2018 & 12 donors & White $(100 \%)$ \\
\hline \multirow[t]{9}{*}{ Education } & $\operatorname{Brazil}^{(19)}$ & 2008 & 737 donors & Some college/higher education (5\%) \\
\hline & Brazil $^{(20)}$ & 2009 & 36 donors & Some college/higher education (36\%) \\
\hline & Brazil $^{(23)}$ & 2010 & 92 donors & Some college/higher education ( $48 \%$ ) \\
\hline & $\mathrm{Brazil}^{(32)}$ & 2016 & 12 donors & Completed high school $(92 \%)$ \\
\hline & China ${ }^{(39)}$ & 2019 & 2680 donors & College/higher education ( $60 \%$ ) \\
\hline & Norway ${ }^{(17)}$ & 2004 & 69 donors & College/higher education (73\%) \\
\hline & Spain $^{(30)}$ & 2015 & 7 donors & College/higher education (majority) \\
\hline & Taiwan ${ }^{(25)}$ & 2013 & 816 donors & College/higher education $(81 \%)$ \\
\hline & $\mathrm{USA}^{(18)}$ & 2007 & 87 donors & College/higher education ( $83 \%$ ) \\
\hline \multirow[t]{9}{*}{ Employment status } & $\operatorname{Brazil}^{(19)}$ & 2008 & 737 donors & Unemployed $(70 \%)$ \\
\hline & Brazil $^{(20)}$ & 2009 & 36 donors & Worked outside the home ( $47 \%$ ) \\
\hline & $\mathrm{Brazil}^{(32)}$ & 2016 & 12 donors & Housewives (42\%) \\
\hline & China ${ }^{(39)}$ & 2019 & 2680 donors & Worked outside the home ( $85 \%$ ) \\
\hline & France $^{(16)}$ & 2003 & 103 donors & Worked outside the home ( $51 \%)$ \\
\hline & Korea ${ }^{(31)}$ & 2016 & 915 donors & Housewives $(62 \%)$ \\
\hline & Spain $^{(30)}$ & 2015 & 7 donors & Worked outside the home (majority) \\
\hline & Taiwan ${ }^{(25)}$ & 2013 & 816 donors & Worked outside the home $(72 \%)$ \\
\hline & $\mathrm{USA}^{(18)}$ & 2007 & 87 donors & Worked outside the home ( $65 \%)$ \\
\hline
\end{tabular}

review identified some differences in donor demographics across geographies, interpretation of this information requires more context related to the local setting.

\section{Donor clinical characteristics}

Birth history frequently included a donor's number of children. Results varied by geographies, with some studies reporting that donors were predominantly primiparous and others predominantly multiparous ${ }^{(16-20,23,25,27-30 \text {, }}$ $32-36,38,39,42)$. The percentage of donors that had preterm births were in the minority in most studies $(8-24 \%)^{(25,27-29,33,39)}$, though two studies in India and Brazil reported the approximately half of donors gave birth pre-term ${ }^{(19,42)}$. Donor birth term could influence the composition of some nutrients in donor milk if donations are made in the first weeks postpartum ${ }^{(45,46)}$, suggesting that this may be useful donor data to regularly collect. Information regarding donors' diseases/conditions ${ }^{(22,33,34)}$ and prenatal clinical care was limited ${ }^{(20,32)}$. Data on characteristics of the donor's child beyond birth term were also scarce. For example, no studies reported the sex of the donor's infant, and only a few studies reported hospitalisation status.

\section{Donor lifestyle characteristics}

There is limited research regarding donors' lifestyle characteristics including diet, exercise, legal and illegal drug use, which does not allow for any type of synthesis 
Table 3 Clinical information about milk bank donors

\begin{tabular}{|c|c|c|c|c|}
\hline Sub-category & Country & Year & Subjects & Finding \\
\hline \multirow[t]{19}{*}{ Birth history } & Brazil $^{(19)}$ & 2008 & 737 donors & Delivered pre-term ( $47 \%)$; had $<3$ children $(94 \%)$ \\
\hline & Brazil $^{(20)}$ & 2009 & 36 donors & Had 1 child $(61 \%)$ \\
\hline & $\operatorname{Brazil}^{(23)}$ & 2010 & 92 donors & Had 1 child $(67 \%)$ \\
\hline & Brazil(32) $^{(32)}$ & 2016 & 12 donors & Primiparous (83\%) \\
\hline & Brazil $^{(34)}$ & 2017 & 3513 donors & Multiparous (94\%) \\
\hline & Brazil $^{(35)}$ & 2017 & $\begin{array}{l}51 \text { donors; } 644 \\
\text { non-donors }\end{array}$ & Donors less likely to have infant in NICU than non-donors \\
\hline & China ${ }^{(39)}$ & 2019 & 2680 donors & Delivered pre-term (8\%) \\
\hline & France $^{(16)}$ & 2003 & 103 donors & Had 1 to 2 children ( $83 \%)$ \\
\hline & India ${ }^{(42)}$ & 2020 & 1553 donors & Delivered pre-term (53\%); multiparous (57\%); infant admitted to NICU (37\%) \\
\hline & Italy ${ }^{(38)}$ & 2018 & 659 donors & Delivered after 35 weeks of gestational age (94\%) \\
\hline & Norway ${ }^{(17)}$ & 2004 & 69 donors & Most donors were primiparous and delivered at term (\% not provided) \\
\hline & Poland $(33)$ & 2017 & 45 donors & Delivered pre-term (24\%) \\
\hline & Spain $(27)$ & 2014 & 63 donors & Delivered pre-term ( $21 \%) ;$ primiparous $(62 \%)$ \\
\hline & Spain $^{(28)}$ & 2014 & 391 donors & Delivered pre-term ( $23 \%)$; primiparous ( $56 \%)$; infant admitted to NICU $(37 \%)$ \\
\hline & Spain ${ }^{(30)}$ & 2015 & 7 donors & Had 1 to 2 children $(100 \%)$ \\
\hline & Spain ${ }^{(29)}$ & 2016 & 36 donors & Delivered pre-term $(17 \%)$ \\
\hline & Taiwan ${ }^{(25)}$ & 2013 & 816 donors & Delivered pre-term ( $8 \%$ ); primiparous ( $69 \%$ ) \\
\hline & $U_{S A}^{(18)}$ & 2007 & 87 donors & Had 1 to 2 children $(80 \%)$ \\
\hline & $\mathrm{USA}^{(36)}$ & 2018 & 12 donors & Primiparous (50\%); had infant in NICU (100\%) \\
\hline \multirow[t]{3}{*}{ Disease } & $\operatorname{Brazil}^{(34)}$ & 2017 & 3513 donors & $\begin{array}{l}\text { HIV prevalence decreased to } 0 \% \text {, syphilis increased to } 1.8 \% \text {, and acute } \\
\text { hepatitis B increased to } 3 \% \text { over } 10 \text { years. }\end{array}$ \\
\hline & Poland ${ }^{(33)}$ & 2017 & 45 donors & Had chronic disease not contraindicated to donation (24\%) \\
\hline & USA ${ }^{(22)}$ & 2010 & 1091 donors & $3.3 \%$ rejected for abnormal serological screening \\
\hline \multirow[t]{2}{*}{ Prenatal care } & $\operatorname{Brazil}^{(20)}$ & 2009 & 36 donors & Attended $3-30$ prenatal healthcare visits $(100 \%)$ \\
\hline & Brazil $^{(32)}$ & 2016 & 12 donors & Attended $7-12$ prenatal healthcare visits $(100 \%)$ \\
\hline
\end{tabular}

NICU, neonatal intensive care unit.

Table 4 Lifestyle characteristic information about milk bank donors

\begin{tabular}{|c|c|c|c|c|}
\hline Sub-category & Country & Year & Subjects & Finding \\
\hline Diet & USA ${ }^{(18)}$ & 2007 & 87 donors & Self-reported always/nearly always eating healthy food ( $56 \%$ ) \\
\hline Exercise & $\mathrm{USA}^{(18)}$ & 2007 & 87 donors & Self-reported exercising $3+$ times/week $(64 \%)$ \\
\hline \multirow{4}{*}{ Legal drug use } & Brazil $^{(23)}$ & 2010 & 92 donors & Self-reported never having smoked ( $82 \%)$ \\
\hline & $U_{S A}^{(18)}$ & 2007 & 87 donors & Self-reported alcohol consumption $<1$ time/month $(77 \%)$ \\
\hline & Spain ${ }^{(27)}$ & 2014 & 63 donors & $\begin{array}{l}\text { Presence of caffeine ( } 45 \% \text { of milk samples); presence of } \\
\text { nicotine }(0.3 \% \text { of milk samples) }\end{array}$ \\
\hline & Spain ${ }^{(29)}$ & 2016 & 36 donors & $\begin{array}{l}\text { Presence of caffeine ( } 50 \% \text { of milk and } 78 \% \text { of hair } \\
\text { samples); presence of nicotine ( } 0 \% \text { of milk and } 3 \% \text { of hair } \\
\text { samples at threshold of active smoker) }\end{array}$ \\
\hline \multirow[t]{2}{*}{ Illegal drug use } & Spain $(27)$ & 2014 & 63 donors & Presence of illegal drugs ( $0 \%$ of milk samples) \\
\hline & Spain ${ }^{(29)}$ & 2016 & 36 donors & $\begin{array}{l}\text { Presence of illegal drugs ( } 0 \% \text { of milk and } 0 \% \text { of hair } \\
\text { samples) }\end{array}$ \\
\hline
\end{tabular}

Table 5 Lactation and breast-feeding experience information about milk bank donors

\begin{tabular}{|c|c|c|c|c|}
\hline Sub-category & Country & Year & Subjects & Finding \\
\hline $\begin{array}{l}\text { Breast-feeding } \\
\text { history }\end{array}$ & $\begin{array}{l}\text { France }^{(16)} \\
\text { USA }^{(36)}\end{array}$ & $\begin{array}{l}2003 \\
2018\end{array}$ & $\begin{array}{l}103 \text { donors } \\
12 \text { donors }\end{array}$ & $\begin{array}{l}\text { Excellent/good breast-feeding experience (97\%); } \\
\text { Exclusive breast-feeding (100\%) }\end{array}$ \\
\hline Clinical support & Brazil(35) & 2017 & $\begin{array}{l}51 \text { donors; } \\
644 \text { non- } \\
\text { donors }\end{array}$ & $\begin{array}{l}\text { Clinical support associated with being a donor included (1) receiving } \\
\text { in-hospital help with breast-feeding and (2) receiving information } \\
\text { about milk expression }\end{array}$ \\
\hline \multirow[t]{2}{*}{$\begin{array}{l}\text { Milk expression } \\
\text { practices }\end{array}$} & Brazil(21) $^{(21)}$ & 2010 & 36 donors & $\begin{array}{l}\text { Expressed manually }(61 \%) \text {; expressed milk } 1+\text { times/d }(72 \%) \text {; factors } \\
\text { influencing expression included beliefs about impact of diet }(47 \%) \text {, availability } \\
\text { of time }(28 \%) \text { and negative emotions }(28 \%) \text {. }\end{array}$ \\
\hline & $U_{S A}^{(18)}$ & 2007 & $\begin{array}{l}87 \text { donors; } \\
19 \text { non-donors }\end{array}$ & $\begin{array}{l}\text { Expressed with personal electrical pump ( } 75 \%) \text {; donors reports fewer problems } \\
\text { with pumping than non-donors }\end{array}$ \\
\hline \multirow[t]{3}{*}{$\begin{array}{l}\text { Beliefs about the } \\
\text { value of milk }\end{array}$} & Brazil $^{(32)}$ & 2016 & 12 donors & $\begin{array}{l}\text { Major theme: importance of breast-feeding for both the baby and the } \\
\text { mother }\end{array}$ \\
\hline & Spain ${ }^{(30)}$ & 2015 & 7 donors & Major theme: benefits of breast-feeding \\
\hline & $U_{S A}^{(26)}$ & 2013 & 19 donors & $\begin{array}{l}\text { Major themes: breast milk being a cure for everything, a gift with expiration } \\
\text { date, majority of middle- and upper-income donors expressed an interest } \\
\text { of receiving compensation }\end{array}$ \\
\hline
\end{tabular}


Table 6 Donor experience information about milk bank donors

\begin{tabular}{|c|c|c|c|c|}
\hline Sub-category & Country & Year & Subjects & Findings \\
\hline \multirow[t]{15}{*}{$\begin{array}{l}\text { Reasons/enablers to } \\
\text { donation }\end{array}$} & $\operatorname{Brazil}^{(19)}$ & 2008 & 737 donors & $\begin{array}{l}\text { Encouraged by a health professional }(61 \%) \text {, received information in the } \\
\text { hospital (50\%) }\end{array}$ \\
\hline & Brazil $^{(20)}$ & 2009 & 36 donors & $\begin{array}{l}\text { Altruism }(92 \%) \text {, excess milk production ( } 61 \%) \text {, to avoid waste }(47 \%) \\
\text { information provided by healthcare professionals and media }(47 \%)\end{array}$ \\
\hline & Brazil(21) & 2010 & 36 donors & Received support from family ( $89 \%)$ and institution (58\%) \\
\hline & Brazil $^{(32)}$ & 2016 & 12 donors & Major themes: altruism, avoid waste, institutional and family support \\
\hline & Brazil( $^{(35)}$ & 2017 & $\begin{array}{l}51 \text { donors; } \\
644 \text { non-donors }\end{array}$ & $\begin{array}{l}\text { Donors were significantly more likely to be encouraged to donate milk at } \\
\text { the hospital than non-donors }\end{array}$ \\
\hline & China ${ }^{(39)}$ & 2019 & 2680 donors & $\begin{array}{l}\text { The internet was the most popular source of information regarding } \\
\text { donations }(33 \%)\end{array}$ \\
\hline & France ${ }^{(16)}$ & 2003 & 103 donors & Having excess milk (57\%) and desire to help others ( $41 \%)$ \\
\hline & Korea ${ }^{(31)}$ & 2016 & 915 donors & Obtained information about donation online ( $76 \%$ ) \\
\hline & Spain $^{(30)}$ & 2015 & 7 donors & $\begin{array}{l}\text { Major themes: information received about milk banks and perceived } \\
\text { approval of family and friends, having excess milk, altruism, empathy, } \\
\text { support from family and milk bank }\end{array}$ \\
\hline & $U_{S A}^{(18)}$ & 2007 & 87 donors & To help others, having excess milk (\% not provided) \\
\hline & $\mathrm{USA}^{(24)}$ & 2012 & 21 donors & $\begin{array}{l}\text { Major themes: physical and emotional meanings of pumping, finding } \\
\text { meaning in perinatal loss, and importance of healthcare providers } \\
\text { addressing lactation with bereaved mothers }\end{array}$ \\
\hline & USA ${ }^{(26)}$ & 2013 & 19 donors & $\begin{array}{l}\text { Major theme: deriving value from the physical and emotional labour of } \\
\text { pumping }\end{array}$ \\
\hline & $U_{S A}^{(36)}$ & 2018 & 12 donors & $\begin{array}{l}\text { Major themes: hope of donation helping others, act of donating was } \\
\text { nurturing for the donor, importance of support from healthcare staff and } \\
\text { desire to share their stories }\end{array}$ \\
\hline & USA $^{(37)}$ & 2018 & 2 donors & $\begin{array}{l}\text { Major themes: milk donation as a mean of processing perinatal loss and } \\
\text { doing something helpful with their milk }\end{array}$ \\
\hline & $\mathrm{USA}^{(43)}$ & 2020 & $\begin{array}{l}95 \text { donor } \\
\text { testimonials }\end{array}$ & Major theme: having excess milk \\
\hline \multirow[t]{5}{*}{ Barriers for donation } & $\operatorname{Brazil}^{(20)}$ & 2009 & 36 donors & $\begin{array}{l}\text { Main reasons to cease donation included returning to work and reduction } \\
\text { in milk production }\end{array}$ \\
\hline & $\operatorname{Brazil}^{(32)}$ & 2016 & 12 donors & Major theme: limited information provided prenatally \\
\hline & Spain $^{(30)}$ & 2015 & 7 donors & $\begin{array}{l}\text { Major themes: lack of healthcare provider knowledge, distance from milk } \\
\text { bank, no support at work and decrease of milk production }\end{array}$ \\
\hline & $U_{S A}^{(18)}$ & 2007 & 87 donors & $\begin{array}{l}\text { Finding time to pump, transporting milk to the bank and problems getting } \\
\text { blood test (\% not provided) }\end{array}$ \\
\hline & USA $^{(37)}$ & 2018 & 2 donors & Major theme: frequent pumping was difficult \\
\hline \multirow[t]{3}{*}{ Donor identity } & $U_{S A}^{(24)}$ & 2012 & 21 donors & $\begin{array}{l}\text { Major themes: identifying as a bereaved mother/grieving the loss of } \\
\text { motherhood }\end{array}$ \\
\hline & USA $^{(40)}$ & 2019 & 80 donors & $\begin{array}{l}\text { Major themes: a temporal donor identity allowed bereaved mothers } \\
\text { opportunity to process loss and reconstruct maternal/female identity }\end{array}$ \\
\hline & $\mathrm{USA}^{(43)}$ & 2020 & $\begin{array}{l}95 \text { donor } \\
\text { testimonials }\end{array}$ & $\begin{array}{l}\text { Major themes: donors had complex and fluid identity including being a } \\
\text { woman, a mother, healthcare professional and prior recipient of milk } \\
\text { donation }\end{array}$ \\
\hline
\end{tabular}

across regions. While milk banks screen donors to ensure they are healthy, lifestyle information could be valuable, as factors associated with maternal diet and lifestyle may influence what is being transferred in the milk.

\section{Lactation and breast-feeding experience}

Donors reported similar beliefs about the importance of breast-feeding and breast milk across three geographies $^{(26,30,32)}$. Donors' beliefs in the value of their milk was only explored in one study, with many donors expressing the desire for compensation. Information about donors' breast-feeding history, clinical support for lactation and milk expression practices was limited to one or two studies, suggesting this is an important area for future research to better understand the donor's path to having excess milk for donation.

\section{Donor Experiences and Beliefs}

The most common donor experience studied was reasons/ enablers for donation ${ }^{(16,18-21,24,26,30-32,35,37,39,43)}$. Common reasons for donation included altruism, having excess milk and avoiding waste $\mathrm{w}^{(16,18,20,30,32,36,37,43)}$. Common enablers for donation were being encouraged to donate and receiving information about milk banks from healthcare providers ${ }^{(19-}$ $21,24,30-32,35,36,39)$. Healthcare providers were reported as a major source of information in Brazil, while online sources were reported as major sources of information in Korea and China ${ }^{(19-21,31,39)}$. Barriers for donation were only assessed in three countries and included finding time to pump, reduced milk production, limited information provided prenatally, returning to work, distance from milk bank and no support at $\operatorname{work}^{(18,20,30,32,37)}$. Qualitative studies that explored donor identity were all conducted in the USA and found that while the act of donating influenced mother's identity, it had a special meaning for bereaved mothers ${ }^{(24,37,40)}$. 
Table 7 Donation pattern information about milk bank donor

\begin{tabular}{|c|c|c|c|c|}
\hline Sub-category & Country & Year & Subjects & Findings \\
\hline \multirow[t]{10}{*}{ Donation volume } & China $^{(39)}$ & 2019 & 2680 donors & 1.9 I (mean) \\
\hline & India ${ }^{(41)}$ & 2019 & $70-4000$ donors & 0.64 I (median) \\
\hline & India ${ }^{(42)}$ & 2020 & 1553 donors & $\begin{array}{l}0.27 \text { I (mean); significantly higher volumes were donated by mothers } \\
\text { with infants in the NICU v. postnatal wards }\end{array}$ \\
\hline & Italy $(38)$ & 2018 & 659 donors & 2.9 I (mean) for term donors and $11.7 \mathrm{I}$ (mean) for pre-term donors \\
\hline & Korea $^{(31)}$ & 2016 & 915 donors & 11.8 I (mean) \\
\hline & Norway ${ }^{(17)}$ & 2004 & 69 donors & 29 I (mean) \\
\hline & Poland (33) & 2017 & 45 donors & $0.65-32$ I (range) \\
\hline & Spain $(28)$ & 2014 & 391 donors & $\begin{array}{l}\text { 3.1 I (median), } 0.04-174 \text { I (range); donation volume was significantly } \\
\text { higher with donors whose infants were hospitalised, had lower } \\
\text { gestational age at birth, lower infant age at time of donation and } \\
\text { were previously milk bank donors }\end{array}$ \\
\hline & Taiwan (25) & 2013 & 816 donors & 17 I (mean) \\
\hline & $U_{S A}^{(18)}$ & 2007 & 87 donors & $30 \mathrm{I}$ (mean) \\
\hline \multirow[t]{6}{*}{ Donor type } & Brazil(20) $^{(20)}$ & 2009 & 36 donors & First-time donors (83\%) \\
\hline & Brazil(32) & 2016 & 12 donors & First-time donors $(92 \%)$ \\
\hline & China $^{(39)}$ & 2019 & 2680 donors & Repeat donors (donated more than three times) $(55 \%)$ \\
\hline & France $^{(16)}$ & 2003 & 103 donors & First-time donors $(72 \%)$ \\
\hline & Korea ${ }^{(31)}$ & 2016 & 915 donors & First-time donors (51\%) \\
\hline & Taiwan ${ }^{(25)}$ & 2013 & 816 donors & First-time donors $(97 \%)$ \\
\hline \multirow[t]{9}{*}{ Milk type } & Brazil( $^{(20)}$ & 2009 & 36 donors & $\begin{array}{l}\text { Started donating within } 3 \text { weeks after delivery (colostrum/transition } \\
\text { milk) }(47 \%)\end{array}$ \\
\hline & $\operatorname{Brazil}^{(23)}$ & 2010 & 92 donors & Majority of donations were mature milk ( $83 \%)$ \\
\hline & China(39) & 2019 & 2680 donors & Started donating after 1 month postpartum (77\%) (mature milk) \\
\hline & Korea $^{(31)}$ & 2016 & 915 donors & $\begin{array}{l}\text { Majority of donations were from } 1 \text { to } 3 \text { months postpartum (mature } \\
\text { milk) }\end{array}$ \\
\hline & Norway ${ }^{(17)}$ & 2004 & 69 donors & $\begin{array}{l}\text { Started donating on average when infant was } 7 \text { weeks old. Range of } \\
\text { infant age at start was } 1-21 \text { weeks (transition and mature milk) }\end{array}$ \\
\hline & Poland ${ }^{(33)}$ & 2017 & 45 donors & $\begin{array}{l}\text { Started donating on average when infant was } 14 \text { weeks old. Range of } \\
\text { infant age at start was } 1-44 \text { weeks (transition and mature milk) }\end{array}$ \\
\hline & Spain $^{(28)}$ & 2014 & 391 donors & $\begin{array}{l}\text { Started donating on average when infant was } 12 \text { weeks old. Range of } \\
\text { infant age at start was } 0-28 \text { months old (colostrum to mature milk) }\end{array}$ \\
\hline & Spain ${ }^{(27)}$ & 2014 & 63 donors & Majority of donations were mature milk (91\%) \\
\hline & Taiwan ${ }^{(25)}$ & 2013 & 816 donors & $\begin{array}{l}\text { Majority of donors (97\%) began donating }>1 \text { month postpartum } \\
\text { (mature milk) }\end{array}$ \\
\hline \multirow[t]{4}{*}{ Donation duration } & $\operatorname{Brazil}^{(20)}$ & 2009 & 36 donors & From 1 to 4 months \\
\hline & Norway ${ }^{(17)}$ & 2004 & 69 donors & From $<1$ to 13 months \\
\hline & Poland ${ }^{(33)}$ & 2017 & 45 donors & From 2-26 weeks \\
\hline & $\mathrm{USA}^{(37)}$ & 2018 & 2 donors & From 6-8 weeks \\
\hline
\end{tabular}

NICU, neonatal intensive care unit.

\section{Donation patterns}

There was a wide range of reported donation volumes per donor (mean or median 0.64-30 1 and range $0 \cdot 04-1741)^{(17,18,25,28,31,33,38,39,41,42)}$. The wide range could be attributed to the differences in milk banking requirements. For example, in Brazil, there is not a minimum donation volume ${ }^{(47)}$, while in the USA some milk banks require a minimum donation of 100 ounces $^{(48)}$. In India and Spain, donors with infants in the NICU/hospitalised provided significantly higher volumes than donors without hospitalised infants ${ }^{(28,42)}$. Donor type was mostly first time ( $v$. repeat) in all regions, although it was not widely reported $^{(16,20,25,31,32)}$. The type of milk commonly donated was mature milk, as the donations started mostly after 1 month postpartum ${ }^{(17,20,25,27,28,31,33,39)}$. This suggests that donors are frequently providing milk that is likely lower in protein than the colostrum and transition milk that would normally be provided by an infant's own mother in the early postpartum period. There was limited information about donation duration (range 2 weeks to 13 months $)^{(17,20,33,37)}$. No studies collected information regarding whether milk bank donors provided their milk elsewhere, including either selling it or sharing with a peer.

\section{Conclusion and future direction}

Although DHM banking continues to grow around the world ${ }^{(49,50)}$, information about the individuals who donate their milk is often limited to a single study per geography, with heterogeneity in the variables reported. Further, onethird of the studies were subject to potential selection bias. Some demographic characteristics were commonly reported across regions, while others, including gender and race, were infrequently explored, suggesting the need to incorporate these demographic variables in future research. Although donors' experiences related to 
donations were frequently reported, enablers and barriers for donation differ among regions studied and not enough is known about what motivates donors to donate. Additionally, factors that could influence the nutritional profile of DHM, including birth timing (term or pre-term), type of milk donated (colostrum, transition or mature), donor diet and infant characteristics, should be more frequently collected. Other factors that have not been widely studied included donor lactation and breast-feeding history, including factors that influence why donors are pumping and amassing surplus milk and donation patterns, including whether milk bank donors are also selling milk to corporations or sharing milk with peers.

\section{Acknowledgements}

Acknowledgements: None. Financial support: This research received no specific grant from any funding agency in the public, commercial or not-for-profit sectors. Conflicts of interest: There are no conflicts of interest. Authorship: M.T.P. conceived of the study; B.G.S. and M.T.P. planned the study; B.G.S. led data collection; B.G.S. and M.T.P. analysed data; B.G.S. wrote the first manuscript draft; B.G.S. and M.T.P. edited the manuscript and agreed on final content. Ethics of human subject participation: Not applicable.

\section{References}

1. Quigley M, Embleton ND \& McGuire W (2019) Formula $v$. donor breast milk for feeding preterm or low birth weight infants. Cochrane Database Syst Rev. Published online: 19 July 2019. doi: 10.1002/14651858.CD002971.pub5.

2. World Health Organization (2019) Donor Human Milk for Low-Birth-Weight Infants. http://www.who.int/elena/titles/ donormilk_infants/en/ (accessed March 2021).

3. Human Milk Banking Association of North America (2021) Homepage. https://www.hmbana.org/ (accessed March 2021).

4. The Brazilian Network of Human Milk Banks (rBHL-Brazil) (2005) Homepage. https://rblh.fiocruz.br/rblh-brasil (accessed March 2021).

5. European Milk Banking Association (2021) Homepage. https://europeanmilkbanking.com/ (accessed March 2021).

6. American Academy of Pediatrics Committee on Nutrition \& Section on Breastfeeding, Committee on Fetus and Newborn (2017) Donor human milk for the high-risk infant: preparation, safety, and usage options in the United States. Pediatrics. Published online: January 2017. doi: 10.1542/ peds.2016-3440.

7. Shenker N, Staff M, Vickers A et al. (2021) Maintaining human milk bank services throughout the COVID-19 pandemic: a global response. Matern Child Nutr. Published online: 6 January 2021. doi: 10.1111/mcn.13131.

8. McCune S \& Perrin MT (2020) Donor human milk use in populations other than the preterm infant: a systematic scoping review. Breastfeed Med 16, 8-20.

9. PATH (2021) Strengthening Human Milk Banking: A Global Implementation Framework. https://www.path. org/programs/maternal-newborn-child-health-and-nutrition/ strengthening-human-milk-banking-resource-toolkit/ (accessed March 2021).

10. Peila C, Moro GE, Bertino E et al. (2016) The effect of Holder pasteurization on nutrients and biologically-active components in donor human milk: a review. Nutrients. Published online: 2 August 2016. doi: 10.3390/nu8080477.

11. Fang MT, Grummer-Strawn L, Maryuningsih Y et al. (2021) Human milk banks: a need for further evidence and guidance. Lancet Glob Health. Published online: February 2021. doi: 10.1016/S2214-109X(20)30468-X.

12. Mills L, Coulter L, Savage E et al. (2019) Macronutrient content of donor milk from a regional human milk bank: variation with donor mother - infant characteristics. $\mathrm{Br} \mathrm{J}$ Nutr 122, 1155-1167.

13. Arksey H \& O'Malley L (2005) Scoping studies: towards a methodological framework. Int J Soc Res Methodol 8, 19-32.

14. Institute of Medicine (1991) Nutrition during Lactation. Washington, DC: National Academies Press.

15. Fincham JE (2008) Response rates and responsiveness for surveys, standards, and the journal. Am J Pharm Educ. Published online: 15 April 2008. doi: 10.5688/aj720243.

16. Azema E \& Callahan S (2003) Breast milk donors in France: a portrait of the typical donor and the utility of milk banking in the French breastfeeding context. J Hum Lact 19, 199-202.

17. Lindemann PC, Foshaugen I \& Lindemann R (2004) Characteristics of breast milk and serology of women donating breast milk to a milk bank. Arch Dis Child Fetal Neonatal Ed 89, F440-F441.

18. Osbaldiston R \& Mingle LA (2007) Characterization of human milk donors. J Hum Lact 23, 350-357.

19. Pimenteira Thomaz AC, Maia Loureiro LV, da Silva Oliveira T et al. (2008) The human milk donation experience: motives, influencing factors, and regular donation. J Hum Lact 24, 69-76.

20. de Alencar LCE \& Seidl EMF (2009) Breast milk donation: women's donor experience. Rev Saude Publica 43, 70-77.

21. de Alencar LCE \& Seidl EMF (2010) Breast milk donation and social support: reports of women donors. Rev Lat Am Enfermagem 18, 381-389.

22. Cohen RS, Xiong SC \& Sakamoto P (2010) Retrospective review of serological testing of potential human milk donors. Arch Dis Child Fetal Neonatal Ed 95, F118-F120.

23. Koyashiki GAK, Paoliello MMB, Matsuo T et al. (2010) Lead levels in milk and blood from donors to the breast milk bank in Southern Brazil. Environ Res 110, 265-271.

24. Welborn JM (2012) The experience of expressing and donating breast milk following a perinatal loss. J Hum Lact 28, 506-510.

25. Chang F-Y, Cheng S-W, Wu T-Z et al. (2013) Characteristics of the first human milk bank in Taiwan. Pediatr Neonatol $\mathbf{5 4}$, 28-33.

26. Pineau MG (2013) Giving milk, buying milk: the influence of mothering ideologies and social class in donor milk banking. In Breastfeeding: Global Practices, Challenges, Maternal and Infant Health Outcomes, pp. 61-76 [T Cassidy, editor]. New York: Nova Science Publishers.

27. Escuder-Vieco D, Garcia-Algar Ó, Pichini S et al. (2014) Validation of a screening questionnaire for a human milk bank to determine the presence of illegal drugs, nicotine, and caffeine. J Pediatr 164, 811-814.

28. Sierra Colomina G, García Lara N, Escuder Vieco D et al. (2014) Profile of human milk bank donors and relationship with the length of the donation. An Pediatr 80, 236-241.

29. Escuder-Vieco D, Garcia-Algar O, Joya X et al. (2016) Breast milk and hair testing to detect illegal drugs, nicotine, and caffeine in donors to a human milk bank. J Hum Lact $\mathbf{3 2}$, 542-545. 
30. Machado RS, Campos Calderón CP, Montoya Juárez R et al. (2015) Experiences of human milk donation in AndalucíaSpain: a qualitative study. Enferm Glob 14, 114-124.

31. Jang HL, Cho JY, Kim M et al. (2016) The experience of human milk banking for 8 years: Korean perspective. J Korean Med Sci 31, 1775-1783.

32. Miranda WD de, Passos MC, Freitas MI de F et al. (2016) Representations of women milk donors on donations for the human milk bank. Cad Saúde Colet 24, 139-144.

33. Barbarska O, Zielińska M, Pawlus B et al. (2017) Characteristics of the regional human milk bank in Poland - donors, recipients and nutritional value of human milk. Rocz Panstw Zakl Hig 68, 395-400.

34. Kupek E \& Savi EO (2017) Milk donor blood screening for HIV, syphilis and hepatitis B markers in a Brazilian human milk bank: prevalence time-trends over the 2005-2015 period. Curr HIV Res 15, 291-296.

35. Meneses TMX de, Oliveira MIC de \& Boccolini CS (2017) Prevalence and factors associated with breast milk donation in banks that receive human milk in primary health care units. J Pediatr 93, 382-388.

36. Candelaria LM, Spatz DL \& Giordano N (2018) Experiences of women who donated human milk.J Obstet Gynecol Neonatal Nurs 47, 556-563.

37. Cole JCM, Schwarz J, Farmer M-C et al. (2018) Facilitating milk donation in the context of perinatal palliative Care. J Obstet Gynecol Neonatal Nurs 47, 564-570.

38. Quitadamo PA, Palumbo G, Cianti L et al. (2018) Might the mothers of premature babies feed them and devote some milk to the milk bank? Int J Pediatr. Published online: 2 December 2018. doi: 10.1155/2018/3628952.

39. Liu X-H, Han S-P, Wei Q-F et al. (2019) The data and characteristics of the human milk banks in mainland China. World J Pediatr 15, 190-197.

40. Oreg A (2019) Milk donation after losing one's baby: adopting a donor identity as a means of coping with loss.
Soc Sci Med. Published online: 27 August 2019. doi: 10. 1016/j.socscimed.2019.112519.

41. Sachdeva RC, Mondkar J, Shanbhag S et al. (2019) A landscape analysis of human milk banks in India. Indian Pediatr 56, 663-668.

42. Nangia S, Ramaswamy VV \& Bhasin M (2020) The profile of donors to a human milk bank in a developing nation. Breastfeed Med 15, 135-139.

43. Oreg A \& Appe S (2020) Ain't no hood like motherhood: the complexity of human milk donor identity. Soc Sci Q 101, 439-458.

44. The New Humanitarian (2018) The Gap in Global Guidelines on Human Milk Banking. https://deeply. thenewhumanitarian.org/malnutrition/articles/2018/08/02/ the-gap-in-global-guidelines-on-human-milk-banking (accessed February 2021).

45. Underwood MA (2013) Human milk for the premature infant. Pediatr Clin North Am 60, 189-207.

46. Gidrewicz DA \& Fenton TR (2014) A systematic review and meta-analysis of the nutrient content of preterm and term breast milk. BMC Pediatr. Published online: 30 August 2014. doi: 10.1186/1471-2431-14-216.

47. The Brazilian Network of Human Milk Banks (rBHL-Brazil) (2005) Human Milk Donation. https://rblh.fiocruz.br/ doacao-de-leite-humano-0 (accessed March 2021).

48. WakeMed Mothers' Milk Bank (2020) Frequently Asked Question. https://www.wakemed.org/care-and-services/womens/supportfor-baby/mothers-milk-bank/faq (accessed March 2021).

49. Israel-Ballard K (2018) Strengthening systems to ensure all infants receive human milk: integrating human milk banking into newborn care and nutrition programming. Breastfeed Med 13, 524-526.

50. Mansen K, Nguyen TT, Nguyen NQ et al. (2021) Strengthening newborn nutrition through establishment of the first human milk bank in Vietnam. J Hum Lact 37, 76-86. 